\title{
New clinically applicable method for visualizing adjacent lung segments using an infrared thoracoscopy system
}

\author{
Noriyuki Misaki, MD, Sung Soo Chang, MD, Hitoshi Igai, MD, Shintarou Tarumi, MD, \\ Masashi Gotoh, MD, PhD, and Hiroyasu Yokomise, MD, PhD
}

\begin{abstract}
Objective: Our objective was to attempt a clinical trial of segmentectomy using the infrared thoracoscopy system after intravenous injection of indocyanine green.
\end{abstract}

\begin{abstract}
Patients and Methods: A total of 8 patients with lung lesions were investigated (5 with primary lung cancer, 2 with metastatic lung tumor, and 1 with inflammatory change). All were scheduled to undergo segmentectomy and had been confirmed to have no allergy to iodine or indocyanine green. Informed consent was obtained from all patients. We identified the dominant pulmonary artery supplying the target segment using reconstructed computed tomography images. The dominant pulmonary artery of the target segment was ligated, and after we had observed the lung using the infrared thoracoscopy system after intravenous injection of indocyanine green $(3.0 \mathrm{mg} / \mathrm{kg})$, and marked the white-to-blue transitional zone by electrocautery, we performed segmentectomy.
\end{abstract}

Results: Average operation time was $150 \pm 62.1$ minutes, and bleeding volume was $68.8 \pm 30.5 \mathrm{~mL}$. Under infrared thoracoscopy, the area with a normal blood supply became stained blue 13 seconds after injection of indocyanine green. Maximum staining intensity was attained 28 seconds after dye injection, and the observation duration was 3.5 minutes. A well-defined color zonation was observed in all patients. We had enough time to mark it. No complications attributable to infrared thoracoscopy after intravenous injection of indocyanine green were encountered.

Conclusions: Infrared thoracoscopy with indocyanine green makes it possible to identify the target lung segment easily and quickly without the need for inflation. This method will be especially useful for cases associated with severe emphysema or when surgery offers a limited view, as is the case with video-assisted thoracic surgery. (J Thorac Cardiovasc Surg 2010;140:752-6)

Lung segmentectomy has been one of the valuable operative methods for general thoracic surgeons. For the surgeon to perform lung segmentectomy, the intersegment line needs to be recognized. Because it is difficult to determine the intersegmental line without any interventions, several previous reports have documented various methods for delineating the intersegmental line for segmentectomy. ${ }^{1-3}$ Some surgeons adopt the inflation and deflation line as the intersegmental line, and others divide the lung parenchyma on the basis of structures such as the pulmonary vessels. A common method is to temporarily reinflate the lung to determine the intersegmental line after the target bronchi are ligated or dissected. ${ }^{1}$ However, it is often difficult to find the intersegmental line in patients with emphysema by this method be-

\footnotetext{
From the Department of General Thoracic Surgery, Breast and Endocrinological Surgery, Faculty of Medicine, Kagawa University, Japan.

Supported by Grants-in-Aid Scientific Research (20890153).

Disclosures: None.

Read at the 90th Annual Meeting of The American Association for Thoracic Surgery, Toronto, Ontario, Canada, May 1-5, 2010.

Received for publication March 25, 2010; revisions received July 1, 2010; accepted for publication July 12, 2010.

Address for reprints: Noriyuki Misaki MD, Second Department of Surgery, Faculty of Medicine, Kagawa University, 1750-1 Ikenobe, Miki-cho, Kita-gun, Kagawa 7610793, Japan (E-mail: nmisaki@med.kagawa-u.ac.jp).

$0022-5223 / \$ 36.00$

Copyright (c) 2010 by The American Association for Thoracic Surgery doi: $10.1016 /$ j.jtcvs. 2010.07 .020
}

cause the lung parenchyma tends to show an advanced state of collateral respiratory structure development, and the emphysematous lungs easily get into an overinflated state. The overinflated lungs offer only limited surgical views, often delaying the procedure and imposing stress on the surgeon.

Using an animal model, we have succeeded in visualizing the segmental line of the lung parenchyma on the basis of the pulmonary arterial blood supply using infrared thoracoscopy (IRT) with indocyanine green (ICG) without any need for lung inflation. ${ }^{4}$ This novel method defines the intersegmental line according to the blood supply. Using IRT with ICG makes it possible to clearly separate the target segment from other areas that show a normal blood flow by eliminating the original blood flow owing to the pulmonary artery ligation. Using IRT with ICG, the transition zone has been proved to correspond to the delineating intersegmental line by means of radiographic and histologic analysis. On the basis of the results we obtained in our preclinical animal study, we applied this technique to the clinical setting and examined the applicability and feasibility of this new method.

\section{MATERIALS AND METHODS Principle of the IRT System \\ The rationale of our IRT system has been reported previously. ${ }^{5}$ In brief, white light from a light source is filtered and converted into infrared light with wavelengths of $940 \mathrm{~nm}$ and $805 \mathrm{~nm}$ (CLV-260SL; Olympus Medical}




\section{Abbreviations and Acronyms \\ $\mathrm{CT}=$ computed tomography \\ $\mathrm{ICG}=$ indocyanine green \\ IRT $=$ infrared thoracoscopy}

Systems Co, Tokyo, Japan), and the lung is illuminated with this infrared light delivered from the tip of a thoracoscope (LTF-Y0006SA41319; Olympus Medical Systems Co). ICG in plasma absorbs light at $805 \mathrm{~nm}$. If the light of both wavelengths is reflected from the lung surface in the absence of ICG, a video processor (CV-260SL; Olympus Medical Systems Co) converts the signal so that the lung is displayed as white. If only $940-\mathrm{nm}$ light is reflected owing to the presence of ICG, the video processor converts the signal so that the lung is displayed as blue.

\section{Patients}

A total of 8 patients were enrolled in this study between November 2008 and October 2009. All patients were planning to undergo an anatomic segmentectomy for tumors and had been checked for allergies to iodine or ICG. Informed consent was obtained from all patients after the risks and benefits of the proposed surgery had been explained by their surgeons. Approval for this study was provided by the Institutional Review Board of the University of Kagawa.

\section{Preoperative Evaluation}

All patients underwent a preoperative workup by computed tomography (CT; Aquillion64, Toshiba Medical Systems Co, Tochigi, Japan) scanning. The CT images were reconstructed 3 dimensionally to evaluate the anatomy of the pulmonary arteries ${ }^{6}$ (Figure 1). The main purpose of the 3-dimensional CT evaluation was to verify whether the target segments were identified by this method. We carefully checked the dominant arteries and bronchi of the target segment preoperatively. By using these 3-dimensional images, we could plan the precise operation procedures and delineate the anatomy of the pulmonary artery that was going to be ligated and resected during the operation.

\section{Surgical Procedure}

This was a pilot study, and it was important to assess the clinical feasibility of this new technique to verify that the correct resected intersegmental line could be found. For this reason, we used the 10- to 12-cm musclesparing anterolateral thoracotomy approach under general anesthesia during single-lung ventilation, which was our standard for segmentectomy.

The dominant pulmonary arteries of the target segment were ligated and divided, and they were identified with the preoperative CT images. ICG (Daiichi Sankyo Co, Ltd, Tokyo, Japan) at $3.0 \mathrm{mg} / \mathrm{kg}$ was rapidly injected into a peripheral vein for observation of the lung under the infrared light. Under the infrared light, the appearance of the lung was clearly separated into 2 areas, white and blue, according to the blood flow on the monitor. The transition zone between the 2 colored areas was then marked on the visceral pleura with electrocautery. Individual ligation or stapling was performed on the corresponding segmental bronchus and on the pulmonary vein as needed. The segmental boundaries were divided with staplers based on the marking on the visceral pleura to the hilar structures. If the tumor margin was not secured during the operation, we performed an extended resection regardless of the marking. We tried to keep the margin at least $1 \mathrm{~cm}$ from the tumor.

\section{RESULTS}

The study patients comprised 6 men and 2 women with a mean age of 74 years (range, 63-84 years). There were 5

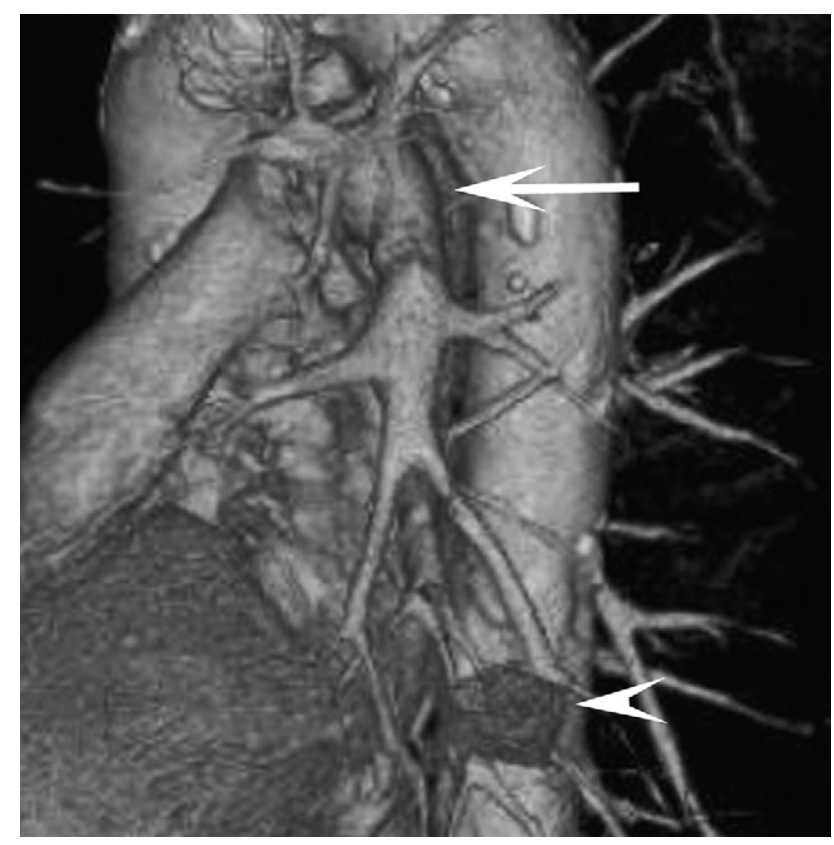

FIGURE 1. The computed tomographic images were reconstructed 3 dimensionally. The left pulmonary artery (arrow) and the tumor (arrowhead) are evident.

primary lung cancers, 2 metastatic lung tumors ( 1 from a primary colon cancer and the other from a primary glomus tumor), and 1 case of inflammatory change. The mean tumor size was $19.9 \mathrm{~mm}$ (range, $8-50 \mathrm{~mm}$ ). The patient characteristics and the target segments are summarized in Table 1.

A well-defined color transition was observed in all patients. Case 3 was a case of primary lung cancer in a man aged 76 years with impaired lung function owing to emphysema. A left basilar segmentectomy was planned. We preserved the left superior pulmonary artery and injected ICG under IRT. The superior segment showed a change to blue, whereas the basilar segment remained white. A transition zone between the 2 colors was clearly depicted. Under the white light, we were unable to detect the 2 adjacent segments (Figure 2). This case required an intervention using mechanical ventilation for 1 night because of hypercapnea attributable to the underlying emphysema. Case 8 was primary lung cancer in a 69-year-old woman who had never smoked. A left upper ventral segmentectomy was planned. We ligated the dominant pulmonary artery of the target segment and then injected ICG under IRT. The lung showed a sharp contrast between the white and blue areas (Figure 3).

For the cases overall, the mean operation time was 150 minutes (range, 94-255 minues), and the mean bleeding volume was $75.4 \mathrm{~mL}$ (range, $40-176 \mathrm{~mL}$ ). With regard to the time during which visualization of color change was possible under IRT, the area with a normal blood supply was stained blue within 13 seconds (range, $8-18$ seconds) after injection of ICG. The maximum staining intensity was 
TABLE 1. Summary of detection of intersegmental line by infrared thoracoscopy

\begin{tabular}{|c|c|c|c|c|c|c|c|c|}
\hline Case No. & Sex & Age (y) & Size (mm) & Histology & Target segment & $\begin{array}{c}\text { Appearance of } \\
\text { staining (s) }\end{array}$ & $\begin{array}{l}\text { Maximum } \\
\text { staining (s) }\end{array}$ & $\begin{array}{l}\text { Observation } \\
\text { duration (s) }\end{array}$ \\
\hline 1 & M & 81 & 50 & Lung ca. & Lingular & 8 & 30 & 187 \\
\hline 2 & M & 79 & 24 & Lung ca. & Rt. apical & 18 & 20 & 270 \\
\hline 3 & M & 76 & 22 & Lung ca. & Lt. basilar & 13 & 30 & 170 \\
\hline 4 & M & 74 & 18 & Lung meta. & Rt. superior & 8 & 32 & 210 \\
\hline 5 & M & 64 & 8 & Lung meta. & Lt. anterior basal & 13 & 33 & 250 \\
\hline 6 & M & 63 & 12 & Inflammation & Lt. lateral basal + posterior basal & 13 & 22 & 180 \\
\hline 7 & $\mathrm{~F}$ & 84 & 15 & Lung ca. & Rt. anterior basal + lateral basal & 16 & 24 & 251 \\
\hline 8 & $\mathrm{~F}$ & 69 & 10 & Lung ca. & Lt. upper ventral & 15 & 30 & 160 \\
\hline
\end{tabular}

attained at 28 seconds (range, 20-33 seconds) after dye injection, and the mean observation duration was 3 minutes 30 seconds (range, 2 minutes 40 seconds-4 minutes $30 \mathrm{sec}$ onds). Emphysematous lung parenchyma was more difficult to deflate than the normal lung, and the former sometimes remained in a semi-inflated state. As a result, the lung tissue tended to stain less than normal. In cases of large tumors, the peripheral parenchyma of the tumors blurred the white and blue boundaries in the early periods. However, even with the large tumors, the stained area showed enough distance from the tumor edges as a clear, distinguished line. No complications were attributed to IRT with ICG.

\section{COMMENT}

When performing lung segmentectomy, it is important for surgeons to find a "true anatomic" intersegmental plane to avoid unnecessary parenchymal resection for preserving maximum pulmonary function ${ }^{2}$ and reducing the incidence of complications such as bleeding and air leakage. ${ }^{7-9}$ This also clarifies the actual margin of the tumor from the intersegmental plane, thus allowing judgment of the need for a combined resection. ${ }^{2,3,7}$ A number of previous reports have advocated specific methods for identifying the intersegmental plane. ${ }^{1-3}$ Although methods based on lung ventilation have several advantages, including their simplicity and the fact that they do not require extra equipment, these approaches require the lung to be inflated to varying degrees, and this occasionally makes it difficult to define the intersegmental line in patients with emphysematous lung. In addition, methods based on the structure of pulmonary vessels require more of the surgeons' experience and surgical skills.

We have developed a new clinically applicable method for visualizing adjacent lung segments based on their blood supply using ICG with IRT. Only the injection of ICG under IRT after ligation of the dominant pulmonary artery allowed for the recognition of the intersegmental line without any lung inflation, and we could maintain clear surgical fields during the operation. It is important to keep an adequate margin from the tumor. One study reported that segmentectomy was not performed for tumors within $1 \mathrm{~cm}$ of the parenchymal margin, ${ }^{1}$ and another reported that the closest segmental margin should exceed $2.0 \mathrm{~cm}$ in cases of primary lung cancer. ${ }^{10}$ It might be better to keep the same state for both the diseased segment and the residual segment to know the exact margins. The distance from the tumor to the intersegmental line is visually evident under a deflated state using this method, and we tried to keep the margin over $1 \mathrm{~cm}$ from the tumor.

There are several limitations to this pilot study. The period of ICG staining is limited, but we were able to mark the

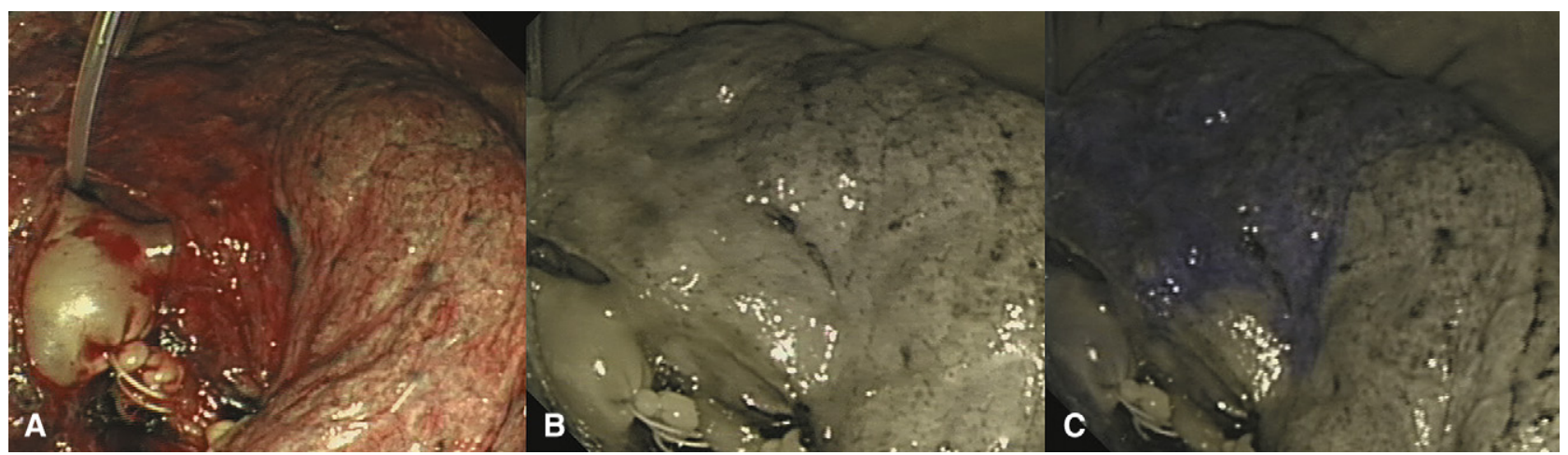

FIGURE 2. Findings of thoracoscopy in case 3. A, The top of the figure represents the cranial direction and the left side the anterior direction. The intersegmental line is not clearly evident under normal light. B, The appearance under infrared light without indocyanine green. C, The intersegmental line is clearly delineated by the boundary of colors under infrared thoracoscopy with indocyanine green. 


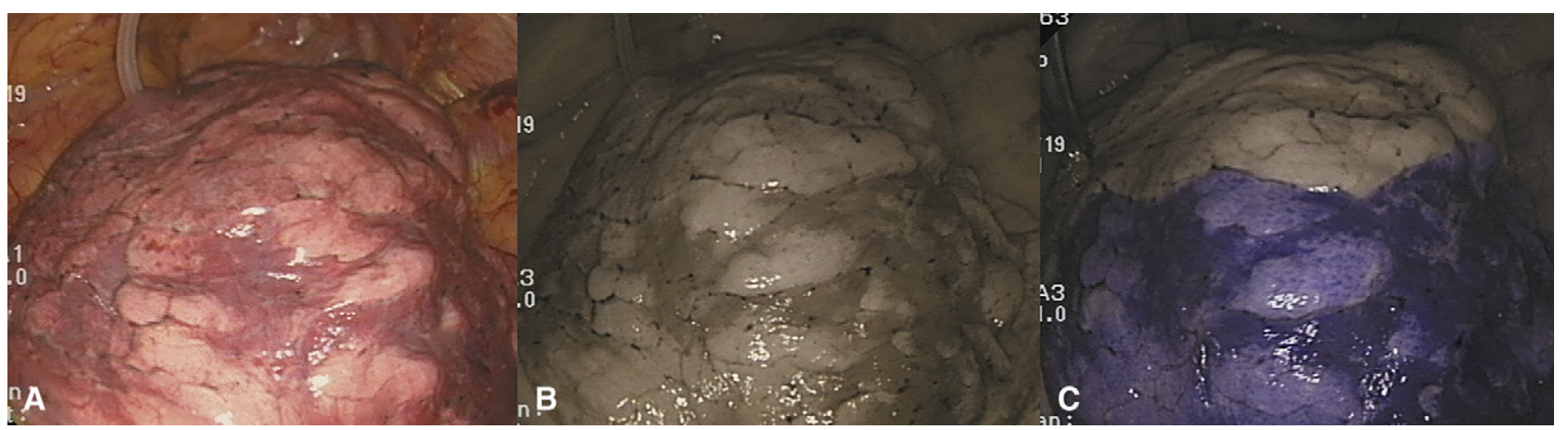

FIGURE 3. Findings of thoracoscopy in case 8 . A, The left upper lobe was found on the left side and the lower lobe on the right. The left basilar pulmonary artery was ligated, and the left superior and lingular pulmonary artery was preserved. B, Appearance under infrared light without indocyanine green. C, The upper lobe and the superior segment in the lower lobe were dyed blue, but the basilar segment remained white under infrared thoracoscopy with indocyanine green.

transitional zone on the visceral pleura without any problems on the basis of the depicted color difference. We had to pay more careful attention in patients with large tumors because, in areas surrounding large tumors, the observation period was shorter than in other areas. Such large tumors may be supplied not only from the segment in which they are located but also from adjacent segments. The doses of ICG that we used for this study were relatively high compared with the recommended dose for general use. We applied ICG at a dose of $3.0 \mathrm{mg} / \mathrm{kg}$ on the basis of our previous experience for identifying lesions in the emphysematous lung. ${ }^{11}$ This dose did not cause any acute or chronic toxicity or side effects attributable to the relatively high-dose ICG injection. After intravenous injection, ICG is known to bind to plasma proteins and lipoproteins. This binding leads to a degree of chemical stabilization of ICG and intravascular confinement. ICG is taken up exclusively by the liver and excreted into the bile within 24 hours. ${ }^{12}$ According to other toxicity studies, the intravenous injection of $5.0 \mathrm{mg} / \mathrm{kg}$ ICG is quite safe and acceptable. ${ }^{13}$ The number of patients in this pilot study was small, but all of the enrolled patients showed similar positive findings with this method, and we attained our objectives for testing this novel approach. For clinical application, the main concerns are the cost and handling of IRT. In this study, we did not have to use any new devices except for the IRT system, and we could perform the operation with the usual equipment. Thus, only the IRT price was a concern; we do not have precise information on the IRT price because we used a prototype scope. Although we used a prototype, the handling of the equipment was not different from the conventional thoracoscope, and our entire faculty and staff could control the IRT without any special training.

In conclusion, for clinical settings, it is acceptable to apply the novel method of IRT with ICG for segmentectomy to identify the intersegmental line. This method will be especially useful for patients who have severe emphysema, in whom there is only a limited view of the intersegmental line, and it would allow surgeons to keep the lung at a de- flated state. Our next objective is to examine the clinical benefit for patients in a randomized clinical trial and to prove the usefulness of this approach for segmentectomy in videoassisted thoracic surgery.

\section{References}

1. Atkins BZ, Harpole DH Jr, Mangum JH, Toloza EM. D‘Amico TA, Burfeind WR Jr. Pulmonary segmentectomy by thoracotomy or thoracoscopy: reduced hospital length of stay with a minimally-invasive approach. Ann Thorac Surg. 2007;84: 1107-13.

2. Okada M, Miura T, Ikegaki J, Katoh H, Itoh H, Tsubota N. A novel video-assisted anatomic segmentectomy technique: selective segmental inflation via bronchofiberoptic jet followed by cautery cutting. J Thorac Cardiovasc Surg. 2006;132: 769-75.

3. Bando T, Miyahara R, Sakai H, Shoji T, Sonobe M, Wada H, et al. A follow-up report on a new method of segmental resection for small-sized early lung cancer. Lung Cancer. 2009;63:58-62.

4. Misaki N, Chang SS, Gotoh M, Yamamoto Y, Satoh K, Yokomise H. A novel method for determining adjacent lung segments with infrared thoracoscopy. J Thorac Cardiovasc Surg. 2009;138:613-8.

5. Gotoh M, Okamoto T, Yamamoto Y, Yokomise H. Real time imaging and quantitative evaluation of the emphysematous lung by infrared thoracoscopy in experimental dogs. ASAIO J. 2005;51:148-51.

6. Nakamoto K, Omori K, Nezu K. Lung Cancer Project Group of West-Seto Inland Sea, Japan. Superselective segmentectomy for deep and small pulmonary nodules under the guidance of three-dimensional reconstructed computed tomographic angiography. Ann Thorac Surg. 2010;89:877-83.

7. Schuchert MJ, Pettiford BL, Pennathur A, Abbas G, Awais O, Landreneau RJ, et al. Anatomic segmentectomy for stage 1 non-small-cell lung cancer: comparison of video-assisted thoracic surgery versus open approach. J Thorac Cardiovasc Surg. 2009;138:1318-25.

8. Schuchert MJ, Pettiford BL, Luketich JD, Landreneau RJ. Parenchymal-sparing resections: why, when, and how. Thorac Surg Clin. 2008;18:93-105.

9. Takagi K, Hata Y, Sasamoto S, Tamaki K, Fukumori K, Shibuya K, et al. Late onset postoperative pulmonary fistula following a pulmonary segmentectomy using electrocautery or a harmonic scalpel. Ann Thorac Cardiovasc Surg. 2010;16:21-5

10. Leshnower BG, Miller DL, Femandez FG, Pickens A, Force SD. Video-assisted thoracoscopic surgery segmentectomy: a safe and effective procedure. Ann Thorac Surg. 2010;89:1571-6.

11. Gotoh M, Yamamoto Y, Igai H, Chang S, Huang C, Yokomise H. Clinical application of infrared thoracoscopy to detect bullous or emphysematous lesions of the lung. J Thorac Cardiovasc Surg. 2007;134:1498-501.

12. Desmettre T, Devoisselle JM, Mordon S. Fluorescence properties and metabolic features of indocyanine green (ICG) as related to angiography. Surv Ophthalmol. 2000;45:15-27.

13. Fox IJ, Wood EH. Indocyanine green: physical and physiologic properties. Mayo Clin Proc. 1960;35:732-44. 


\section{Discussion}

Dr Federico Venuta (Rome, Italy). I congratulate the authors. Because I did not receive the paper in advance, my comments will be based on the abstract and the presentation.

I have just a couple of points. Did you perform this study through a thoracoscope or was it through a standard thoracotomy?

Dr Misaki. We performed segmentectomy by the open method. All patients underwent open segmentectomy. We used IRT only for observation.

Dr Venuta. According to your study, this navigation system should avoid the need for reinflation of the lung, and this is the only potential advantage. What are the costs of this system and are there any other fields of surgery in which this device can be used so that the costs can be shared with other specialties?
Dr Misaki. The cost, I cannot answer now. I do not know.

Dr Keshavjee. Dr Yokomise, do you have any information on the cost of the system in your center?

Dr Yokomise. This is supported by Olympus and there is no cost for me now. So we must study and Olympus will probably sell the machine in the near future. This time there is no cost. Regarding the specific field, you mean another field?

Dr Venuta. Yes. For example, liver surgery.

Dr Yokomise. Yes, in my institute the collaboration between abdominal surgery, especially for liver resection, and probably like a SPY system (Novadaq Technologies, Bonita Springs, Fla) for heart surgery to look at the coronary arteries. We can probably use this machine for abdominal surgery as well as heart surgery, as in our clinical experience. 